\title{
What might be known: Epistemic modality and uncertain contexts
}

\author{
Laurent Roussarie \\ Université Paris 8 \& UMR 7023, CNRS
}

\section{Introduction}

Epistemic modals relativize the truth value of a proposition to some body of knowledge. It is thus reasonable to consider that their truth conditions concern at least as much the attitude of the speaker(s) towards the proposition as the content of the proposition itself. Epistemic modals can be seen as evidential comments on propositions (von Fintel and Gillies 2007). Veltman (1996), Groenendijk et al. (1996) and von Fintel and Gillies (2007) have shown that this particular contribution is better accounted for within a dynamic semantic implementation than in terms of more traditional (i.e. static) truth conditions.

Albeit insightful, the dynamic formalization of epistemic modals causes a double problem. First declarative sentences containing an epistemic operator such as the one in (1) become non-informative $e^{1}$ _even though they are defined as meaningful.

(1) Hitch might be the murderer.

This alleged lack of informativeness is due to the fact that the relevant body of knowledge with respect to which the epistemic is interpreted is assimilated to the information that constitutes the common ground. The dynamic meaning of (1) merely amounts to test the compatibility of the proposition 'Hitch is the murderer' with the common ground, but it does not add any new information to it.

Second, questions containing an epistemic modal (henceforth EMQ for $e$ pistemically modalized questions) as the one in (2) fail to be truly interrogative.

(2) Might Hitch be the murderer?

The dynamic formalization improperly predicts that EMQs will not be construed as real request for information, as they cannot be interpreted in a context where their

This work has been undertaken as part of the programme "Sentence typing", of the Fédération Typologie et Universaux Linguistiques (TUL: FR 2559) of the CNRS. I want to thank Hans-Georg Obenauer, Hans-Martin Gärtner, Andreas Haida, Philippe Schlenker, Fatima Hamlaoui, Patrica Cabredo Hofherr, Bridget Copley and Lea Nash for their help, discussion, suggestions and/or reviews. I would also like to thank an anonymous reviewer and the audience at SALT 19 for their helpful comments, and especially David Beaver who fruitfully pointed out to me the connection between my proposal and his 1997 work.

${ }^{1}$ See also Dekker (2009) on this issue. 
answer is not already known by the speaker. ${ }^{2}$

The solution proposed here follows Beaver's (1997) idea that a realistic implementation of dynamic semantics should view the participants in a conversation as having uncertain knowledge of the information they share. Therefore a realistic modelization of the context should involve a set of alternative common grounds. From this assumption, it is possible to define the dynamic contribution of epistemically modalized declaratives as genuinely informative. Moreover the present proposal also allows to define an explanatory semantics for EMQs as questions about the speakers' knowledge rather than about the actual state of the world.

This paper is structured as follows. Section 2, recalls the main lines of the standard static and dynamic semantics of epistemic modals, and presents the whys and wherefores of the formal issues, namely the lack of informativeness of declaratives and the lack of inquisitiveness of questions. Section 3 lays out the proposal: a modification of the dynamic semantics of epistemic modality so as to solve the above mentioned issues mainly on a formal basis. The last section discusses some more concrete motivations for the present formalization and proposes that EMQs' semantics can be worked out by making a connection with Kratzer's $(1981,1991)$ notion of ordering source.

\section{Formal issues}

\subsection{Epistemic modality in assertions}

Let's begin with a simple reminder of the canonical analysis of modals due to Kratzer $(1981,1991)$. A modal sentence is analyzed as a quantification over possible worlds. The truth-conditions of a possibility and of a necessity are given in (3). The nuclear scope $(\varphi)$ of the quantification is the propositional material to which the modal expression applies, the so called prejacent proposition; and the restriction or modal base $(B)$ is the set of all the worlds that are accessible from the world of evaluation $w$. A possibility amounts to a consistency condition between the modal base and the (intension of the) prejacent (3a); a necessity amounts to a relation of entailment from the modal base to the prejacent (3b).

$$
\begin{aligned}
& \text { a. } \llbracket \diamond(B)(\varphi) \rrbracket^{w}=1 \text { iff } \llbracket B \rrbracket^{w} \cap \llbracket \varphi \rrbracket \neq \varnothing \\
& \text { b. } \llbracket \square(B)(\varphi) \rrbracket^{w}=1 \text { iff } \llbracket B \rrbracket^{w} \subset \llbracket \varphi \rrbracket
\end{aligned}
$$

In the case of epistemic modality, the modal base represents a body of "established knowledge for a group of people, a community etc." (Kratzer 1981: p. 45). Accordingly the sentence in (1) is true in a world $w$ iff the proposition that Hitch is the murderer is consistent with what is known in $w$. Here I represent the modal base with the variable $B$ whose denotation depends on $w$ as, by definition, it is the

\footnotetext{
${ }^{2}$ Although it is sometimes assumed that the epistemic reading of a modal is not (or barely) available in questions (Jackendoff 1972, Drubig 2001, a.o.), von Fintel and Iatridou (2003) have however argued that EMQs are well formed and interpretable.
} 
conjunction (or intersection) of only true propositions, and of course what counts as a true proposition is relative to the worlds in which the truth value is evaluated. $B$ also behaves as a more conventional variable whose value depends on the context: the relevant body of knowledge on which an epistemic modal is based is what is known by the participants of the conversation in a particular context of utterance. This is precisely one of the insights of the dynamic implementation of epistemic modality.

In dynamic semantics the notion of context plays a central role; the dynamic meaning of an expression is not defined in terms of its truth conditions but as its Context Change Potential or CCP. I adopt here a framework of Groendijk, Stokhof and Veltman's Update Semantics (Veltman 1996, Groenendijk et al. 1996) which is fittingly designed to treat epistemic modality. Formally the CCP of an expression is defined as a relation (or a function) from contexts onto contexts; it will be noted as $\llbracket \cdot \rrbracket^{\text {ccp }}$ with a usual postfix notation. A context is implemented as an information state $s$, viz. a set of possibilities or-for the sake of simplification-a set of possible worlds. Thus the application of the CCP of $\alpha$ to an input context $s_{i}$ is written $s_{i} \llbracket \alpha \rrbracket^{\text {ccp}}$; it yields an output context $s_{o}$ and the whole operation is called the update of $s_{i}$ by $\alpha$. By way of illustration the CCP of an asserted sentence is the usual intersective and eliminative operation (Stalnaker 1978):

$$
s \llbracket \varphi \rrbracket^{\mathrm{ccp}}=s \cap \llbracket \varphi \rrbracket=\left\{w \in s \mid \llbracket \varphi \rrbracket^{w}=1\right\}
$$

The initial state $s$ is updated by eliminating the worlds that do not belong to the intension of $\varphi$. As an information state is a set of alternatives of what can count as being the actual world in view of what the speakers know, the process of eliminating worlds amounts to a growth of information. Hence informativity is directly correlated to the eliminative character of the CCP: $\varphi$ is informative in $s$ if it is strictly eliminative, and $\varphi$ is strictly eliminative in $s$ iff $s \llbracket \varphi \rrbracket^{\text {ccp }} \subsetneq s$.

A context, in the form of an information state, thus corresponds to a body of knowledge; it is a mere implementation of Stalnaker's (1978) common ground-or, more precisely, of his context set, which is the intersection of the propositions composing the common ground. Such an information state is also clearly reminiscent of the notion of epistemic modal base. That is why a very natural move in dynamic semantics, when it came to implement the semantics of epistemic modalities (Veltman 1996, Groenendijk et al. 1996, von Fintel and Gillies 2007), was to assimilate contextual information states with contextual epistemic modal bases. Consequently the CCP of epistemic sentences is defined as follows, where the state $s$ plays both roles:

$$
\begin{aligned}
& \text { a. } s \llbracket \diamond \varphi \rrbracket^{\mathrm{ccp}}=\left\{w \in s \mid s \llbracket \varphi \rrbracket^{\mathrm{ccp}} \neq \varnothing\right\}=\left\{\begin{array}{l}
s \text { if } s \cap \llbracket \varphi \rrbracket \neq \varnothing \\
\varnothing \text { otherwise }
\end{array}\right. \\
& \text { b. } s \llbracket \square \varphi \rrbracket^{\mathrm{ccp}}=\left\{w \in s \mid s \llbracket \varphi \rrbracket^{\mathrm{ccp}}=s\right\}=\left\{\begin{array}{l}
s \text { if } s \subset \llbracket \varphi \rrbracket \\
\varnothing \text { otherwise }
\end{array}\right.
\end{aligned}
$$

If we consider that obtaining the empty state $\varnothing$ amounts to a failure of the course of the conversation, then (5) can be understood as saying that the update of 
any state $s$ by $\diamond \varphi$ or $\square \varphi$ succeeds just in case $\varphi$ is consistent with or follows from $s$ respectively. But a successful update yields an output state $s$ which is identical to the input state. This means that the CCP of a modal sentence is simply a test, that is a function which evaluates the coherence and the appropriateness of the context without altering it. This is supported by the idea (as in Veltman (1996) for instance) that an epistemic modal sentence does not genuinely express a proposition, as it does not convey information about how the world is but rather about how the speakers' knowledge is. In short, (5) says that epistemic sentences do not contribute to the growth of information in the conversation; they are never strictly eliminative. At best when $\diamond \varphi$ is asserted the speakers will only remember that the context at hand is consistent with $\varphi$.

However the assertion an epistemic modal sentence can arguably add some information in the common ground of a conversation. Take for instance the case of criminal investigations: a detective who acknowledges the assertion of (1) (or who draws by himself the conclusion that (1)) will be happy to eliminate the options in which it is not possible that Hitch is the murderer. In this way, epistemic modal sentences are informative.

\subsection{Epistemic modality in questions}

Now let's turn to questions, in which the problem is somewhat more serious. A common view on the (static) semantics of questions (Groenendijk and Stokhof 1984 ) is that in any world $w$ a question denotes a proposition which is the meaning of its true (and complete) answer in $w$. Hence the meaning of a question is defined as a function from $\mathscr{W}$ to $\wp(\mathscr{W})$, or equivalently as a binary relation on $\mathscr{W}$. It is an equivalence relation which connects together all the worlds in which the question has the same answer.

$$
\llbracket ? \varphi \rrbracket=\left\{\left\langle w, w^{\prime}\right\rangle \in \mathscr{W} \times \mathscr{W} \mid \llbracket \varphi \rrbracket^{w^{\prime}}=\llbracket \varphi \rrbracket^{w}\right\}
$$

As an equivalence relation, the meaning of a question forms a partition on $\mathscr{W}$ dividing it into the different possible propositional answers. On this basis we can define an important property of questions in context, viz. the notion of inquisitiveness (Groenendijk 1999):

(7) $\quad ? \varphi$ is inquisitive w.r.t $s$ iff there exist $w_{1}$ and $w_{2} \in s$ such that $\left\langle w_{1}, w_{2}\right\rangle \notin \llbracket ? \varphi \rrbracket$, (i.e. such that $\llbracket \varphi \rrbracket^{w_{1}} \neq \llbracket \varphi \rrbracket^{w_{2}}$ ).

The definition says that a question is inquisitive in a state $s$ if it actually divides it into several parts. Put differently: a question is inquisitive in $s$ if its answer is informative in $s$. This characterizes the fact that the speaker doesn't know the answer and is really seeking it. So inquisitiveness can be taken as a felicity condition as long as the question has to be interpreted as a real request for information.

The meaning of an EMQ would then straightforwardly follow from (6) in a compositional manner:

$$
\llbracket ? \diamond \varphi \rrbracket=\left\{\left\langle w, w^{\prime}\right\rangle \in \mathscr{W} \times \mathscr{W} \mid \llbracket \diamond \varphi \rrbracket^{w^{\prime}}=\llbracket \diamond \varphi \rrbracket^{w}\right\}
$$


Note that a first concern with this definition is that, contrary to what has been mentioned above with respect to the dynamic analysis, it assumes the existence and relevance of the "modal" propositions $\llbracket \diamond \varphi \rrbracket$ and $\llbracket \neg \diamond \varphi \rrbracket$, for they are the two possible answers sorted out by the relation $\llbracket ? \diamond \varphi \rrbracket$. But let's not give up yet; the intension of an epistemic modal sentence can still (at least theoretically) be worked out on the basis of the more classical (and static) truth conditions as given in (3). The proposition $\llbracket \diamond \varphi \rrbracket$ (or $\llbracket \diamond(B)(\varphi) \rrbracket$ ) is the set of worlds that yield an epistemic modal base which is consistent with $\varphi$. A simple way to grasp this intension is to think of the modal bases in terms of the traditional accessibility relations of modal logic. Indeed the epistemic modal base $\llbracket B \rrbracket^{w}$ is the set of all worlds which are epistemically accessible to $w$. Let's call $R_{\mathcal{E}}$ this epistemic accessibility relation. Then $\llbracket \diamond \varphi \rrbracket$ is the set of all worlds that are $R_{\mathcal{E}}$-accessible to some world belonging to $\llbracket \varphi \rrbracket$ :

$$
\llbracket \diamond \varphi \rrbracket=\left\{w \in \mathscr{W} \mid \exists w^{\prime} \in \llbracket \varphi \rrbracket \text { and } w^{\prime} R_{\varepsilon} w\right\} .
$$

It is worth noting that an epistemic accessibility relation is commonly assumed to be transitive and euclidean. ${ }^{3}$ As a consequence all the worlds that belong to a given epistemic modal base are connected together by $R_{\mathcal{\varepsilon}}$. And this also applies for any information state since it represents a body of knowledge just in the same way as an epistemic modal base.

(10) For any information state $s, \forall w, w^{\prime} \in s, w R_{\varepsilon} w^{\prime}$.

Now the problem with the semantics of EMQs is the following. Suppose a speaker $A$ asks (2) as a genuine request for information. This means that $A$ does not know whether Hitch might or might not be the murderer, namely that $? \diamond \varphi$ is (or should be) inquisitive with respect to her personal information state. But because this state is based upon an epistemic transitive relation, this inquisitive requirement leads to a contradiction.

\section{(2) A: Might Hitch be the murderer?}

The demonstration goes as follows. Let $s_{A}$ be the speaker's epistemic information state, and suppose that $? \diamond \varphi$ is inquisitive with respect to $s_{A}$. By definition (7) this means that there exist $w_{1}$ and $w_{2} \in s_{A}$ such that $\diamond \varphi$ is true in $w_{1}$ and $\diamond \varphi$ is false in $w_{2}$. So $s_{A}$ is consistent with $\diamond \varphi$ and $\neg \diamond \varphi$. Next as $\diamond \varphi$ is true in $w_{1}$, then according to (9) there exists $w_{3}$ such that $w_{1} R_{\varepsilon} w_{3}$ and $\varphi$ is true in $w_{3}$. Since $w_{3}$ is accessible to $w_{1}$, according to (10) $w_{3}$ is accessible to every world in $s_{A}$. In other words, every world in $s_{A}$ is accessible to a $\varphi$-world; this simply means that $\diamond \varphi$ is true in every world of $s_{A}$. But this also implies that $\neg \diamond \varphi$ is true in no world of $s_{A}$. Hence a contradiction: for the sake of inquisitiveness we have just assumed

\footnotetext{
${ }^{3} R_{\varepsilon}$ is transitive iff $u R_{\varepsilon} v$ and $v R_{\varepsilon} w$ entails $u R_{\varepsilon} w$; it is euclidean iff $u R_{\varepsilon} v$ and $u R_{\varepsilon} w$ entails $v R_{\varepsilon} w$. These properties are commonly required, in order to account respectively for the speaker's positive and negative introspection (that is when a speaker knows that $\varphi$, she knows that she knows it; and when she doesn't know whether $\varphi$, she knows that she doesn't know it. Furthermore $R_{\mathcal{E}}$ is actually an equivalence relation (being reflexive and symmetric as well).
} 
that $\neg \diamond \varphi$ is true in $w_{2}$. In short, the rationale is that if $\varphi$ is possible in a world $w$ belonging to $s$ then it is possible in every world of $s$ and therefore $\diamond \varphi$ is necessarily true with respect to $s$ and the answer of $? \diamond \varphi$ is already known.

The problem with this analysis of EMQs is that the model leaves no room to express an agent's lack of epistemic commitment towards a modal sentence. In the classical picture the ignorance of $\psi$ is equivalent to the knowledge of $\diamond \psi$ (and of $\diamond \neg \psi){ }^{4}$ So to ignore $\diamond \varphi$ is to know that $\diamond \diamond \varphi$; and as long as we are dealing with only one $R_{\varepsilon}$ (or only one epistemic information state), $\diamond \diamond \varphi$ is equivalent to $\diamond \varphi \ldots$ We could escape the contradiction by allowing different epistemic operators $\diamond$, which in fact would amount to consider several different $R_{\varepsilon}$ or epistemic states. This is precisely the basic idea of the proposal I present in what follows.

\section{Proposal}

\subsection{Structuring the context}

In brief what we need here is to define the CCP of a modal sentence in such a way that it can be strictly eliminative with respect to some contexts. As a consequence we will be able to conceive some contexts consistent with a modal sentence and its negation. This will automatically give us the inquisitiveness of the corresponding EMQ.

Now what kind of things can be eliminated by these CCPs? It cannot be possible worlds because a modal sentence does not "examine" worlds one by one, it only deals with them globally within a state (or a modal base). Then a solution will be to discriminate between several states in a given context. Formally it amounts to evaluating epistemics, and any other expression, with respect $\mathrm{s}$ to $a$ set of information states. This implies a modification of the implementation and the conception of the notion of a conversational context: it is now defined as a set of sets of possibilities (or worlds). Let's call such a set an information space. Such an implementation is advocated in Beaver $(1997)^{5}$ in order to provide a realistic view of the context, i.e. the common ground. Indeed it is most reasonable to assume that the participants of the conversation do not have a precise and clear cut view of their common ground; their knowledge of what they share can only be hypothetical and therefore uncertain. Technically this means that they bear a stock of alternative common grounds. This is directly rendered by sets of states, i.e. information spaces.

The new definition of the CCPs of modal sentences can thus be formulated recursively as functions from information spaces onto information spaces:

\section{(11) Let $S$ be a set of information states $(S \subset \wp(\mathscr{W}))$ :}

\footnotetext{
${ }^{4}$ This is by the way directly entailed by the principles of classical epistemic modal logic where the "knowing" operator is exactly the epistemic necessity $\square$. Therefore "not knowing" is $\neg \square$ which is the same as $\diamond \neg$.

${ }^{5}$ Beaver calls the sets of states information sets. As sets are omnipresent in the formalism, I suggest to make use of another term here, viz. information spaces.
} 


$$
\begin{aligned}
& \text { a. } S \llbracket \diamond \varphi \rrbracket^{\mathrm{ccp}}=\left\{s \in S \mid s \llbracket \diamond \varphi \rrbracket^{\mathrm{ccp}}=s\right\}=\{s \in S \mid s \cap \llbracket \varphi \rrbracket \neq \varnothing\} \\
& \text { b. } S \llbracket \square \varphi \rrbracket^{\mathrm{ccp}}=\left\{s \in S \mid s \llbracket \square \varphi \rrbracket^{\mathrm{ccp}}=s\right\}=\{s \in S \mid s \subset \llbracket \varphi \rrbracket\}
\end{aligned}
$$

The CCP of a possibility discards all the states that are not consistent with the prejacent proposition; and the CCP of a necessity discards all the states that do not entail the prejacent. Consequently an epistemic modal sentence can now be strictly eliminative (i.e. informative): some states, and eventually some possibilities, can be eliminated from the context. That solves (at least technically) our first problem.

Note that it is also easy to give a general definition of the CCP for any sentence (modal or not) with respect to information spaces. The principle-as presented in Beaver (1997) — is the following: in order to update a space $S$ with $\psi$, take every state in $S$, update it with $\psi$ and collect the output state in a set which will constitute the output space:

$$
S \llbracket \psi \rrbracket^{\mathrm{ccp}}=\left\{s^{\prime} \in \mathscr{W} \mid \exists s \in S, s \llbracket \psi \rrbracket^{\mathrm{ccp}}=s^{\prime}\right\}
$$

The present proposal also allows to settle our inquisitiveness problem with modal questions. Indeed within an information space $S$ a question like $? \diamond \varphi$ can sort out the states with respect to which $\varphi$ is or is not possible. Thus for $? \diamond \varphi$ to be inquisitive in $S, S$ must contain at least a state consistent with $\varphi$ and a state inconsistent with $\varphi$ :

$? \diamond \varphi$ is inquisitive in $S$ if $\exists s_{1}, s_{2} \in S$ s.t. $s_{1} \cap \llbracket \varphi \rrbracket \neq \varnothing$ and $s_{2} \cap \llbracket \varphi \rrbracket=\varnothing$.

We will come back to the semantics EMQ in section 4.3, but before that let's look at some formal implications of the introduction of information spaces in the formalism.

\subsection{Consistency and support}

In dynamic semantics the notions of truth and entailment do not play as central a role as in classical static semantics. What is at stake with dynamics is the transfer of information and the semantic relationship between utterances and contexts. This is captured by two significant notions: consistency and support (Groenendijk et al. 1996). They are defined as follows:

a. $\varphi$ is consistent with $s$ iff $s \llbracket \varphi \rrbracket^{\mathrm{ccp}}$ exists and $s \llbracket \varphi \rrbracket^{\mathrm{ccp}} \neq \varnothing$.

b. $\varphi$ is supported by $s$ iff $s \llbracket \varphi \rrbracket^{\mathrm{ccp}}$ exists and $s \llbracket \varphi \rrbracket^{\mathrm{ccp}}=s$.

The notion of consistency is related to possibility and the notion of support to necessity: (14) merely says that $\varphi$ is consistent with $s$ iff $\varphi$ is possible in $s$, and $s$ supports $\varphi$ iff $\varphi$ is necessary in $s$. These two notions also qualify the information exchange. A consistent sentence prevents an update failure. Thus an assertion will be informative and acceptable for an addressee if it is consistent with his information state. On the other hand if a sentence is supported by a state, it will keep it intact; this is what enables speaker's sincerity. So for a speaker, an assertion will 
be honest and reliable if it is supported by her information state. It means that she speaks the truth or at least that she is certain of what she says.

On the basis of the new definitions of CCPs, one can define the two notions with respect to information spaces in a very similar and straightforward way:

a. $\varphi$ is consistent with $S$ iff $S \llbracket \varphi \rrbracket^{\text {ccp }}$ exists and $S \llbracket \varphi \rrbracket^{\text {ccp }} \neq \varnothing$.

b. $\varphi$ is supported by $S$ iff $S \llbracket \varphi \rrbracket^{\text {ccp }}$ exists and $S \llbracket \varphi \rrbracket^{\text {ccp }}=S$.

But one can also define a notion in between consistency and support. Let's call it weak or minimal support:

(16) a. $\varphi$ is minimally supported by $S$ iff $S \llbracket \varphi \rrbracket^{\text {ccp }}$ exists and there is at least an $s \in S$ s.t. $s \in S \llbracket \varphi \rrbracket^{\text {ccp }}$.

This notion of minimal support corresponds to the cases where at least one state in the input information space remains intact after the update. It is weaker than normal support, because it does not require the whole space to remain unchanged. It entails consistency but it is more specific, because the output space yielded by a consistent sentence may only contain states that are smaller than those in the input space. This notion renders the idea that a speaker may assert a sentence which is certain with respect to some of her information states, but not all. Actually, this may be what we do all the time: when speaking and asserting, we rarely summon all our knowledge. .

Likewise, it is possible to define variants of consistency, because there are many ways by which the output space can be non empty. For instance (16b) defines strong or maximal consistency; it says that in order to be maximally consistent with $S, \varphi$ must be consistent with every state in the input space. Thus to be maximally consistent with $S$ is to be maximally informative in $S$. But it is also a property of sentences whose truth completely "hangs in the balance" in $S$. This would correspond to pure and unbiased possibility.

(16) b. $\varphi$ is maximally consistent with $S$ iff $S \llbracket \varphi \rrbracket^{\text {ccp }}$ exists and for every $s \in$ $S \llbracket \varphi \rrbracket^{\mathrm{ccp}}, s \llbracket \varphi \rrbracket^{\mathrm{ccp}} \neq \varnothing$.

Note that these notions are also connected to inquisitiveness: in general a question ? $\psi$ is inquisitive in $S$ if $\psi$ is consistent with $S$. And when the question contains an epistemic modal, the condition are more demanding: following the definitions above, ? $\diamond \varphi$ is inquisitive in $S$ iff the prejacent $\varphi$ is consistent but not maximally consistent with $S^{6}$.

\footnotetext{
${ }^{6}$ In this paper I do not really focus on questions with a necessity operator; but for the sake of completeness, let's just mention that a ? $\square \varphi$ question is inquisitive in $S$ iff $\varphi$ is minimally supported but not supported by $S$.
} 


\section{Consequences}

\subsection{Motivation for information spaces}

First I want to discuss the conceptual connection between information spaces and the familiar notion of common ground. In Update Semantics, information states are usually taken to play the role of the context set generated by the common ground. As an information space involves several states, one might be tempted to conclude that the formalization proposed in the previous section implies that several common grounds are in play in order to interpret epistemic modals. This is not a desirable outcome; it is more reasonable to consider that a conversation is normally interpreted with respect to one common ground, that is one context. Actually the insight that underlies the notion of information spaces is that they are merely a way to add more structure to the common ground/context set. Formally this means that here the common ground $(C G)$ remains the classical basic notion as defined by Stalnaker, it is the set of all presuppositions shared by the interlocutors; the context set $(c)$ is its global intersection (i.e. $c=\bigcap C G$ ) and the corresponding information space $(S)$ is a part of the power set of $c$ (i.e. $S \subseteq \wp(c)$ and $c=\bigcup S$ ). Thus the context set is still to be seen as a sort of epistemic information state shared by the interlocutorsbut only "a sort of" because it does not correspond to one accessibility relation. The genuine epistemic information states are those which compose the information space, and each one is generated by a different accessibility relation.

What should be investigated now is the significance of these multiple accessibility relations. In other words: What do information spaces, as sets of states, stand for? and how are they motivated?

A first way to answer this is what could be called the "multi-agent perspective". After all, the present discussion is about the interpretation of questions and therefore pertains to dialogical interactions. So a natural move should be to bring the addressee into play. This idea can be supported by the fact that sometimes a spontaneous way to construe an EMQ consists in "completing" it with some epistemic frame setter as in (17).

(17) According to you/in your opinion, might Hitch be the murderer?

These "according to you", "in your opinion" etc. seem to refer merely to the addressee's personal epistemic modal base-some mundane variants of "in view of what you know". Therefore the information requested in (17) would turn out to be about a consistency test concerning the addressee's modal base, without specifically involving the speaker's modal base.

Thus a simple motivation for information spaces is to consider that each information state composing the space comes from a participant in the conversation. Besides, this view is reminiscent of other related works. For instance, in order to model the notion of bias Gunlogson (2001) defines the context set as the union of the speaker's and addressee's public beliefs-which actually corresponds to two information states. As for the specific issue of the interpretation of epistemic modality, Stephenson (2007) argues that the epistemic modal base that has 
to be taken into account is relative to a judge parameter; this implies that the context should contain as many epistemic states as there are judges. A similar idea is advocated by von Fintel and Gillies (2008) according to whom an epistemic modal (might) sentence has to be evaluated with respect to a "cloud" of contexts, that is states delimited by some groups of speakers and/or addressees.

Of course an accurate modelization of the context has to bring the addressee (and other participants) into the picture. But I think that it will not completely settle the present issue. For an important feature of the context against which an EMQ is normally uttered is (at least is some cases) that the speaker herself ignores whether $\varphi$ is possible or not-even when she is asking for the addressee's opinion as in (17). This means that we have to ascribe her some uncertain knowledge by directly assigning her several information states.

\subsection{Dealing with uncertain information}

In the remainder of this paper I propose to tackle the problem just from the speaker's stance (and to leave open the question of the precise formalization of multi-agent contexts).

An information space can be seen as a disjunction of information states. Among them some are "better" than others. A better state is a more realistic one, that is, a state which is more likely to contain the actual world. An EMQ is thus a quest for the good (or the best) states proposed in the context, and ? $\diamond \varphi$ is construed as "are the good (or best) states (i.e. modal bases) consistent with $\varphi$ ?". Moreover as an epistemic modal base is usually assimilated to some available evidence, ${ }^{7}$ this can also be put in terms of a quest for evidence. I therefore suggest that the meaning of a question with epistemic modality as (2) can be made explicit along these terms:

Might Hitch be the murderer?

$=$ "Is the good available evidence consistent with the proposition 'Hitch is the murderer'?"

The important point here is that a piece of evidence is a proposition which is present in the context (that's why it is available and worth considering by the speakers) but which must not belong to the common ground. Indeed the common gound contains only true propositions (or propositions that are taken for granted by the interlocutors) and we have seen that when $? \diamond \varphi$ is uttered the interlocutors already know that $\varphi$ is consistent with every proposition of the common ground (because if it were not the case, then $\varphi$ would be known to be false in the context and $? \diamond \varphi$ would not be inquisitive). So the evidence which is questioned in (18) corresponds to propositions whose truth value is not known in the context and only have the status of assumptions.

This semantics for modal questions can be worked out by making a another connection with Kratzer's $(1981,1991)$ analysis of modalities-namely with the

\footnotetext{
${ }^{7}$ Indeed along Kratzer's (1991: p. 639) lines, a sentence such as "Hitch might be the murderer" unfolds as "In view of the available evidence, Hitch might be the murderer."
} 
notion of ordering source. An ordering source is a set of propositions provided by the context which is used to organize the worlds within the modal base. It enhances the analysis of modals as it enables it to account for graded modal forces, to solve some logical problems with non realistic modal bases (e.g. counterfactuals, deontic/samaritan paradox...), and especially to take into account some more or less reliable information in addition to what is supplied by the epistemic modal base. This is precisely what is at stake in our suggested semantics for EMQs (18): the pieces of evidence are more or less reliable propositions which do not follow from the modal base but are involved in the computation of the modals' truth conditions.

The main outlines of the formalization are the following. An ordering source $O$ induces an order $\leq_{o}$ among the worlds of any modal base: $w \leq_{o} w^{\prime}$ means that $w$ is closer to the ideal formed by $O$ than $w^{\prime}$ (roughly in the cases where $w$ satisfies more propositions in $O$ than $w^{\prime}$ ). It is then always possible to define the (sub)set of worlds in $\llbracket B \rrbracket^{w}$ that come closest to $\cap O$; let's call it $\min _{\leq_{o}}\left(\llbracket B \rrbracket^{w}\right)$ and define it as $\min _{\leq_{o}}\left(\llbracket B \rrbracket^{w}\right)=\left\{w^{\prime} \in \llbracket B \rrbracket^{w} \mid \forall w^{\prime \prime} \in \llbracket B \rrbracket^{w}\right.$, if $w^{\prime \prime} \leq_{o} w^{\prime}$ then $\left.w^{\prime} \leq_{o} w^{\prime \prime}\right\}$. This way the modal quantification becomes more restricted: we no longer quantify over the whole modal base $B$ but only over a subset of it, viz. $\min _{\leq_{o}}\left(\llbracket B \rrbracket^{w}\right)$ :

a. $\llbracket \diamond(B, O)(\varphi) \rrbracket^{w}=1$ iff $\min _{\leq_{o}}\left(\llbracket B \rrbracket^{w}\right) \cap \llbracket \varphi \rrbracket \neq \varnothing$

b. $\llbracket \square(B, O)(\varphi) \rrbracket^{w}=1$ iff $\min _{\leq_{o}}\left(\llbracket B \rrbracket^{w}\right) \subset \llbracket \varphi \rrbracket$

Usually modals are ambiguous because they can refer to different modal bases but also to different ordering sources. As for epistemics, given one modal base $B$ (i.e. what is known), if we consider different ordering sources $\mathrm{O}_{1}, \mathrm{O}_{2}$, $\mathrm{O}_{3}$.. (i.e. what can be assumed), we'll get several epistemic information states, viz. $\min _{\leq_{o 1}}(B), \min _{\leq_{o 2}}(B), \min _{\leq_{o 3}}(B) \ldots$ (i.e. what might be known). This is nothing more than a way to structure the epistemic modal base (or context set) as an information space.

\subsection{Complex possibilities}

In this section I propose a formal implementation à la Update Semantics of this role of ordering sources in the dynamic interpretation of epistemic modality. Then I will show how it can provide an explanatory formalization of the semantics of EMQs'.

Up to now, I have presented a rather simple formalization of information states and possibilities. An information state is a set of possibilities, and as a first approximation a possibility has been equated to a possible world $w$. But actually, in Update Semantics (Groenendijk et al. 1996), possibilities are more complex objects: they have to encode information about the world but also information about the discourse. In order to do this, assignments need to be added to possibilities. Thus a possibility is rather defined as a tuple $\langle w, g\rangle$ where $g$ is an assignment; $g$ describes a possible state of the record of discourse referents available at this point in the discourse. ${ }^{8}$

\footnotetext{
${ }^{8}$ This is a rough simplification as in Groenendijk et al. (1996) the discourse information is in fact
} 
I propose to add another parameter to each possibility: a (possibly empty) set of propositions $o$, which will provide an ordering source worth examining in the context. Thus a possibility is defined as a tuple $\langle w, o, g\rangle$. Let's call them complex possibilities; information states are now sets of complex possibilities, and I will notate them as $\sigma$. A $\sigma$-state implicitly covers the traditional notion of context set: for a given $\sigma$ the underlying context set is merely the set of all worlds $w$ such that $w$ appears in a possibility of $\sigma$. In $\sigma$, worlds are somehow weighted by one or several ordering sources. Roughly speaking being a possibility in $\sigma$, i.e. $\langle w, o, g\rangle \in \sigma$, means that "according to the assumptions in $o, w$ is a good candidate to be the actual world."9

I will consider that the order $\leq{ }_{o}$, induced by any $O$, also applies to complex possibilities, in the same way it applies to worlds alone in the original definition. That is for any ordering source $O:\left\langle w_{1}, o_{1}, g_{1}\right\rangle \leq_{o}\left\langle w_{2}, o_{2}, g_{2}\right\rangle$ iff $w_{1} \leq_{o} w_{2}$. The ordering of two possibilities with respect to $O$ only depends on $O$ and the possibilities' worlds, but not on the ordering sources stored in the possibilities. Accordingly, for any state $\sigma$ and any ordering source $O, \min _{\leq_{o}}(\sigma)$ is then defined just as in section 4.2:

$$
\min _{\leq_{o}}(\sigma)=\left\{i \in \sigma \mid \forall i^{\prime} \in \sigma, \text { if } i^{\prime} \leq_{o} i \text { then } i \leq_{o} i^{\prime}\right\}
$$

The main idea is that the CCP of a modal sentence is meant to dismiss the ordering sources rather than worlds. For instance in the case of an epistemic possibility $\diamond \varphi$ its CCP is defined in a compositional and inductive manner as: ${ }^{10}$

$$
\sigma \llbracket \diamond \varphi \rrbracket^{\mathrm{ccp}}=\left\{\langle w, o, g\rangle \in \sigma \mid \min _{\leq_{o}}(\sigma) \llbracket \varphi \rrbracket^{\mathrm{ccp}} \neq \varnothing\right\}
$$

Thus (21) mainly tests the ordering sources of $\sigma$ and dismisses those which cause a failure of the consistency condition with $\varphi$. For the sake of homogeneity with the other notations used in this paper, the definition can also be put in terms of the static meaning of $\varphi$, which here is noted $\llbracket \varphi \rrbracket^{g}$ :

implemented by means of two components: a function $g$ and a referent system $r$. See (Groenendijk et al. 1996) for the details of the formalization. Here I just want to emphasize on the fact that an element of a context contains at least information about the world $(w)$ and information about the discourse $(g)$.

${ }^{9}$ Here, unlike in Kratzer $(1981,1991)$, the ordering sources are not functions from worlds to sets of propositions, but merely sets of propositions. However in the present formalization each $o$ occurs in a possibility $\langle w, o, g\rangle$ and thus is indexed by a world $w$. The functional dependence between $o$ and $w$ is therefore still present here. Moreover the $\sigma$-states allows us to consider several "natures" of ordering sources and not only several values for a given ordering source, since a world $w$ can be associated with different $o$ 's. By "nature" I mean to refer to the particular kinds of information an ordering source is dedicated to provide, for instance a description of the normal course of events, some hearsay claims, or some open assumptions introduced earlier in the discourse, etc.; whereas the value refers to the precise propositions that constitute, in a given world, the content of the provided information.

${ }^{10}$ The CCP of an epistemic necessity could be defined along the same lines, as something like: $\sigma \llbracket \square \varphi \rrbracket^{\text {ccp }}=\left\{\langle w, o, g\rangle \in \sigma \mid \min _{\leq_{o}}(\sigma) \llbracket \varphi \rrbracket^{\text {ccp }}=\min _{\leq_{o}}(\sigma)\right\}$. But actually it wouldn't be completely accurate because the update by $\varphi$ may modify the " $g$ "-parameters in the output state, for instance if a new discourse referent is introduced in $\varphi$ by means of an indefinite noun phrase. Hence the CCP must rather be defined in terms of subsistence of the input state. See Groenendijk et al. (1996) for details on this notion. 


$$
\sigma \llbracket \diamond \varphi \rrbracket^{\mathrm{ccp}}=\left\{\langle w, o, g\rangle \in \sigma \mid \min _{\leq_{o}}(\sigma) \cap \llbracket \varphi \rrbracket^{g} \neq \varnothing\right\} .
$$

Contrary to the definition in (5), the update defined in (21) does not perform just one test but as many as there are different ordering sources $o$ in $\sigma$. Some tests may succeed, some others may fail; this is how some possibilities may be discarded from the context. If a possibility $\langle w, o, g\rangle$ is discarded because $o$ fails the consistency test, it does not necessarily mean that $w$ disappears from the context; indeed $w$ may still occur in other possibilities (with other $o$ 's). However if every ordering source that is associated to a given world $w$ within a possibility has been discarded, then ipso facto $w$ is eliminated from the information state. In this way a modal sentence can really be informative since it can really shrink the context set. But of course it is not as strongly eliminative as a non-modal sentence. With respect to the $\sigma$-states, the CCP of a non-modal sentence directly eliminates possibilities in the usual way, on the basis of a satisfaction condition, ignoring the ordering sources stored in $\sigma$ :

$$
\sigma \llbracket \varphi \rrbracket^{\mathrm{ccp}}=\left\{\langle w, o, g\rangle \in \sigma \mid \llbracket \varphi \rrbracket^{w, g}=1\right\}
$$

We can now put together the semantics of EMQs. The contribution of a question is to raise an issue in the context. Technically it amounts to create a partition on the current information state or, equivalently, to add a relation on the possibilities composing the state. The relational meaning of a question can therefore be seen as an operation on the contextual information state, viz. a function of $\sigma$ yielding a set of pairs of possibilities from $\sigma \times \sigma$. I will not take it to be the real CCP of a question as the output of the function is not of the same type as the input ${ }^{11}$ and I will note it simply as $\llbracket ? \varphi \rrbracket^{\sigma}$ :

$$
\llbracket ? \varphi \rrbracket^{\sigma}=\left\{\left\langle\langle w, o, g\rangle,\left\langle w^{\prime}, o^{\prime}, g\right\rangle\right\rangle \in \sigma \times \sigma \mid \llbracket \varphi \rrbracket^{w, g}=\llbracket \varphi \rrbracket^{w^{\prime}, g}\right\}
$$

As in (6), $\llbracket ? \varphi \rrbracket^{\sigma}$ sorts the possibilities in $\sigma$ by connecting together those with respect to which $? \varphi$ has the same answer. Now the relational meaning of a EMQ follows from (21) and (22): it connects together the possibilities according to their ordering sources. The partition divides $\sigma$ into two sets: on the one hand the possibilities whose ordering sources give a $\min _{\leq_{o}}(\sigma)$ consistent with $\varphi$ and on the other hand those whose ordering sources fail the consistency test with $\varphi$ :

$$
\begin{aligned}
& \llbracket ? \diamond \varphi \rrbracket^{\sigma}=\left\{\left\langle\langle w, o, g\rangle,\left\langle w^{\prime}, o^{\prime}, g\right\rangle\right\rangle \in \sigma \times \sigma \mid \min _{\leq_{o}}(\sigma) \cap \llbracket \varphi \rrbracket^{g} \neq \varnothing \Leftrightarrow\right. \\
& \left.\min _{\leq_{o^{\prime}}}(\sigma) \cap \llbracket \varphi \rrbracket^{g} \neq \varnothing\right\}^{12}
\end{aligned}
$$

\footnotetext{
${ }^{11}$ A more accurate way to define the $\mathrm{CCP}$ of a question is to design the context directly as a relation between possibilities, i.e. a set of pairs of possibilities. Thus the CCP of a question disconnects possibilities whereas the CCP of an assertion eliminates possibilities. See Groenendijk (1999).

$12 \mathrm{~S}$. Kaufmann suggested (p.c.) a simpler formalization by considering $o$ as a function from worlds to sets of propositions. Roughly this yields the following definition (with simple information states $s): s \llbracket \diamond \varphi \rrbracket^{\text {ccp }}=\left\{w \in s \mid \min _{\leq_{o(w)}}(s) \llbracket \varphi \rrbracket^{\text {ccp }} \neq \varnothing\right\}$. The CCP discards the worlds $w$ such that $o(w)$ fails the consistency test. According to this implementation, we are dealing with only one parameter $o$; it is obviously more in accordance with Kratzer's formalization. However my proposal is more general - and perhaps more expressive - as it accounts for the different natures of ordering sources and for the possible ambiguity of epistemic modals; see footnote 9.
} 
The crucial feature of this definition is that the sorting carried out by $? \diamond \varphi$ basically pertains to the different ordering sources occurring in $\sigma$. This implies that it is not impossible to find two possibilities sharing the same world, $\langle w, o, g\rangle$ and $\left\langle w, o^{\prime}, g^{\prime}\right\rangle$, that are not connected by $\llbracket ? \diamond \varphi \rrbracket^{\sigma}$; in other words a same world $w$ may appear in different (and contradictory) answers. This is because, as mentioned above, an EMQ is not really a question about how is the world but mainly about how is the context. Indeed (25) can be glossed by the somehow tortuous "do the good ordering sources available in the context pick some known facts that are consistent with $\varphi$ ?". But actually this gloss is nothing more than a detailed version of what is suggested in (18), namely: "is the good available evidence consistent with $\varphi$ ?".

\section{Conclusion}

According to the dynamic semantics of epistemic modality presented in this paper, declarative modal sentences can be made informative and EMQs can be formally interpreted, as long as the conversational context (common ground) is modelized as a body of uncertain knowledge. Technically this implies that the context must be structured as a set of organized possibilities, for instance by assigning them some propositions which are used as ordering sources. This formalization is based on the idea that there are several ways to store information in the context: on the one hand the common ground includes the known facts (taken for granted by the interlocutors), and on the other hand a set of ordering sources gathers some less reliable assumptions at stake in the discourse or the conversation. A significant consequence of the analysis is that EMQs appear to be questions about the context, rather than about the state of the world. This is a peculiar feature, since it is usually assumed that knowledge of the context is a prior requirement to determine the meaning of an expression. ${ }^{13}$

Among the issues demanding future work on EMQs, the most important is the analysis of constituent questions-as this paper only focuses on yes/no questions. Constituent EMQs probably demand a non-trivial adjustment of the present account. Besides, their analysis may shed interesting light on a special (non-standard) category of interrogative sentences described e.g. by Obenauer (2006) as "can'tfind-the-value" questions. This category is identified on the basis of formal (syntactic) properties, especially in various Romance languages. These questions sometimes display a particular affinity with epistemic modal operators as in (26).

Where (the hell) can the murderer be hidden?!

A "can't-find-the-value" question is a real request for information, but it also conveys - maybe on an expressive dimension - that the speaker has already asked herself the question and hasn't succeeded in finding any answer. This is not unrelated to the specificity of EMQs presented here. Indeed when asking an

\footnotetext{
${ }^{13}$ This point is probably not unrelated to the idea advocated by von Fintel and Gillies (2008) that might sentences are ambiguous by design.
} 
EMQ, the speaker overtly confesses that her epistemic information state is uncertain; in a way she signals that she "doesn't know exactly what she knows" (or, if one assimilates information spaces to sets of doxastic modal bases, then she signals that she doesn't know what to believe). This may be a way to formalize the speaker's perplexity that is normally expressed via a "can't-find-the-value" question.

Another extension worth examining is to investigate the double role of modal declaratives. A modal sentence does not only provide information, it also opens some new possibilities to be considered in the subsequent discourse (as in modal subordination for instance). Thus it creates a hypothetical common ground. This contribution can be implemented dynamically by adding in the contextual $\sigma$-state a new ordering source containing the prejacent proposition.

\section{References}

Beaver, David I.: 1997, Presupposition and Assertion in Dynamic Semantics, Studies in Logic, Language and Information. CSLI Publications, Stanford, CA.

Dekker, Paul: 2009, 'A notion of epistemic might with explanatory value', in Journées Sémantique et Modélisation (JSM 2009). Paris.

Drubig, Hans B.: 2001, 'On the syntactic form of epistemic modality'. Ms., University of Tbingen.

von Fintel, Kai and Anthony S. Gillies: 2007, 'An opinionated guide to epistemic modality', in T. S. Gendler and J. Hawthorne (eds.), Oxford Studies in Epistemology 2, 32-62. Oxford University Press, New York.

von Fintel, Kai and Anthony S. Gillies: 2008, 'Might made right'. Ms. MIT and University of Michigan, to appear in a volume on epistemic modality, edited by A. Egan and B. Weatherson, Oxford University Press.

von Fintel, Kai and Sabine Iatridou: 2003, 'Epistemic Containment', Linguistic Inquiry 34, 173-198.

Groenendijk, Jeroen: 1999, 'The logic of interrogation: Classical version', in T. Matthews and D. Strolovitch (eds.), Proceedings of Semantics and Linguistic Theory (SALT) IX, 109-126. Cornell University Press, Ithaca.

Groenendijk, Jeroen and Martin Stokhof: 1984, Studies on the Semantics of Questions and the Pragmatics of Answers, Doctoral dissertation, University of Amsterdam.

Groenendijk, Jeroen, Martin Stokhof, and Frank Veltman: 1996, 'Coreference and modality', in S. Lappin (ed.), Handbook of Contemporary Semantic Theory, 179-216. Blackwell, Oxford.

Gunlogson, Christine: 2001, True to Form: Rising and Falling Declaratives as Questions in English, Doctoral Dissertation, University of California Santa Cruz.

Jackendoff, Ray: 1972, Semantic Interpretation in Generative Grammar. The MIT Press, Cambridge.

Kratzer, Angelika: 1981, 'The notional category of modality', in H.-J. Eikmeyer 
and H. Rieser (eds.), Words, Worlds, and Contexts. New Approaches to Word Semantics, 38-74. Walter de Gruyter \& Co., Berlin.

Kratzer, Angelika: 1991, 'Modality', in A. von Stechow and D. Wunderlich (eds.), Semantik/Semantics. An International Handbook of Contemporary Research, 639-650. Walter de Gruyter, Berlin-New York.

Obenauer, Hans-Georg: 2006, 'Special interrogatives - Left periphery, whdoubling, and (apparently) optional elements', in J. Doetjes and P. Gonzalves (eds.), Romance Languages and Linguistic Theory 2004 - Selected Papers from Going Romance 2004, 247-273. John Benjamins, Amsterdam/Philadelphia.

Stalnaker, Robert C.: 1978, 'Assertion', in P. Cole (ed.), Pragmatics (Syntax and Semantics 9), 315-332. Academic Press, New York.

Stephenson, Tamina: 2007, 'Judge dependence, epistemic modals, and predicates of personal taste', Linguistics \& Philosophy 30, 487-525.

Veltman, Frank: 1996, 'Defaults in Update Semantics', Journal of Philosophical Logic 25, 221-261. 